\title{
Review of: "Selective targeting of the TLR2/MyD88/NF-kB pathway reduces $\alpha$-synuclein spreading in vitro and in vivo"
}

\author{
Yihong $\mathrm{Ye}^{1}$ \\ 1 National Institutes of Health
}

Potential competing interests: The author(s) declared that no potential competing interests exist.

Cell-to-cell transmission of misfolding-prone $\alpha$-synuclein ( $\alpha$-Syn) is a key pathological event in Parkinson's disease (PD). Preformed $\alpha$-Syn fibrils (PFF) injected into mouse brain can be transmitted from the injection site to other areas of the brain, causing pathological features similar to those observed in patient brains. $\alpha$-Syn PFF can also act on microglial cells, inducing an inflammatory state via Toll like receptor 2 (TLR2). These two processes were considered independent of each other, contributing to PD pathology in a parallel manner. The study by Dutta reveals an unexpected link between these two processes. Specifically, the authors show that $\alpha$-Syn PFF induced TLR2/MyD88-dependent NFkB activation in microglial cells, activating the secretion of cytokines such as IL1-beta and TNF, which can act on neurons to induce $\alpha$-Syn expression. As a result, the propagation of injected $\alpha$-Syn PFF is significantly enhanced, which causes neurodegeneration. Importantly, the authors previously reported a peptide that is capable of disrupting the TLR2 and MyD88 interaction. When they administrated this peptide to their PD mice via a nasal route, the peptide can alleviate PFF-induced gliosis and reduce the propagation of $\alpha$-Syn PFF and the associated disease phenotypes.

The study is very thoroughly designed with each point addressed by multiple approaches. A major attraction is the demonstration of a pharmacological administration route and a TLR2 specific inhibitory peptide, which when combined, may offer a new therapeutic strategy for PD patients. While I find this paper interesting, there are several thoughts that I wish can be clarified by the authors.

1. The TLR2 inhibitory peptide does not seem to be very potent when tested in cultured cells (Figure 1). The authors used 10uM to treat cells. Do they know the IC50 of the peptide in vitro? When given to the animal, how much peptide can reach the brain? Have they analyzed the PD and PK of the peptide in CSF? Answers to these question will be essential to gauge the therapeutic potential of this strategy.

2. It is well known that TLR2/MyD88 activation also activates NFkB. In this regard, why do the authors think that the action of $\alpha-S y n$ PFF in vivo need to go through microglial cells? Can't PFF just activate neurons directly via TLR2/NFkB to upregulate $\alpha$-Syn?

3. Along the same line, why neurons respond to IL1beta and TNFalpha by activating $\alpha$-Syn? What is the 
underlying cellular logic? There are many single cell transcriptome studies on PD, which were published. It would be helpful if the authors can compare the notes to see if upregulation of $\alpha$-Syn mRNA was observed in other PD transcriptome studies. For summary of these studies, see file:///Users/yihongy/Downloads/biomedicines-09-00368.pdf

4. There are several errors in the paper. Fig. $4 a, b$ were never mentioned in the paper. Supplementary Fig. 14c mentioned on page 12 does not exist. The figure legends could certainly include more information to make them easy to read. 